\title{
Safety and efficacy of polycaprolactone copolymer nanosphere hydrogel injected into the scalp dermal tissue of rats
}

\author{
Seung Jun Lee', Woo Sung Lee ${ }^{2}$, \\ Chul Hoon Chung ${ }^{1}$ \\ ${ }^{1}$ Department of Plastic and \\ Reconstructive Surgery, Hallym \\ University College of Medicine, Seoul; \\ ${ }^{2}$ Rose Plastic Surgery Clinic and Rose \\ Lab, Seoul, Korea
}

\begin{abstract}
Background Currently, dermal fillers need to be $25 \mu \mathrm{m}$ or larger to reduce in vivo degradation by macrophages. However, the large size of fillers may cause side effects, including interruption of blood flow and nodule formation. Therefore, using rats, we tested a polycaprolactone copolymer hydrogel with nanoscale particles that could maintain a low in vivo degradation rate.

Methods Thirty-six 6-week-old Sprague-Dawley rats were divided into group A (normal saline), group $B$ (polycaprolactone microsphere filler), and group C (polycaprolactone copolymer nanosphere hydrogel). The corresponding materials were injected into the dermal layer of the scalp of the rats. At 4, 8, and 12 weeks after injection, blood biochemical and kidney and liver histological analyses were performed. Tissues were examined using hematoxylin-eosin staining to observe tissue infiltration of materials. Collagen formation in the dermal tissue of the scalp was observed with Masson trichrome staining and the collagen content was quantified using a soluble collagen assay kit. Results The histologic examination for organ infiltration showed no abnormal findings. All blood test results were within the normal ranges. The amount of collagen at 12 weeks increased by $1.22 \mathrm{mg} / \mathrm{g}$ in group C and by $0.6 \mathrm{mg} / \mathrm{g}$ in group B.

Conclusions The results reveal that the nanosphere complex near the injection site induced collagen formation. Regardless of the sphere size, aggregation of the copolymer prevented macrophage phagocytosis. The polycaprolactone copolymer nanosphere hydrogel was effective for more than 3 months when injected in the scalp dermal tissue of Sprague-Dawley rats and can be used safely.
\end{abstract}

Keywords Polycaprolactone / Nanosphere / Dermal fillers
This work was supported by the Technology Development Program (S2608849) funded by the Ministry of SMEs and Startups (MSS, Korea).

\section{INTRODUCTION}

Plastic surgery has advanced significantly over the past few decades. There is an increasing demand for noninvasive cosmetic plastic

Received: Apr 30, 2019 Revised: Jun 23, 2019 Accepted: Aug 19, 2019

Correspondence: Chul Hoon Chung Department of Plastic and

Reconstructive Surgery, Hallym University College of Medicine, 150

Seongan-ro, Gangdong-gu, Seoul 05355, Korea

Tel: +82-2-2224-2246, Fax: +82-2-448-0114, E-mail: c21ps@hanmail.net

Copyright @ 2019 The Korean Society for Aesthetic Plastic Surgery.

This is an Open Access article distributed under the terms of the Creative Commons Attribution Non-Commercial License (http://creativecommons.org/licenses/by-nc/4.0/) which permits unrestricted non-commercial use, distribution, and reproduction in any medium, provided the original work is properly cited. www.e-aaps.org surgery because it causes less pain and scarring, speeds recovery, and reduces the incidence of post-surgical complications, such as adhesions and wound rupture.

Several noninvasive cosmetic plastic surgery procedures, including botulinum toxin injections, dermal fillers, cog threads, ultrasounds, and lasers, are performed increasingly frequently. For example, the therapeutic range of dermal filler has been gradually expanding as new materials, methods, and applications are continuously being introduced. The materials currently used for dermal fillers include biodegradable hyaluronic acid (HA) and biodegradable collagen stimulators, such as calcium-hydroxyapatite, poly-Llactic acid, and polycaprolactone (PCL). Additionally, nonbiodegradable products such as polymethylmethacrylate are also being used. Biodegradable collagen stimulators are the most recently in- 
troduced material; they induce neocollagenesis, which increases the volume at the injection site [1-3].

Dermal fillers are used for soft tissue augmentation and restoration of deep wrinkles. Recently, a tendency has emerged to use fillers to improve skin texture by inducing skin moisturization, revascularization, and collagen synthesis [4-6].

Currently, PCL-based dermal fillers are popular among polymer fillers [7]. PCL dermal filler is sphere-shaped, with an average particle size of $25-50 \mu \mathrm{m}$, and consists of 70\% carboxymethyl carbonate (CMC) and 30\% PCL. PCL is a non-toxic biocompatible and biodegradable polymer that has been widely used in bone engineering, medical devices, and drug delivery systems [8-10].

All currently used fillers are composed of microspheres ranging from $25 \mu \mathrm{m}$ to $50 \mu \mathrm{m}$ in size. At this particle size, the microspheres or particulates avoid being phagocytosed by macrophages, enabling them to exert a persistent effect [11]. However, this particle size can induce many side effects, including interruption of blood flow and formation of nodules in the surrounding tissues, due to vascular obstruction or mass effects $[12,13]$. The size also makes it difficult to inject fillers into the dermal layer of a dense tissue.

For this reason, we hypothesized that reducing the size of the particle to the nano-sized biocompatible and biodegradable copolymer hydrogel developed by Rose Lab would reduce the disadvantages of larger fillers. Biodegradable copolymers are cost-effective, do not evoke a strong immune response, and remain in the dermal tissue for a longer period than HA fillers [14].

Therefore, in this study, a PCL copolymer nanoparticle hydrogel (PCL-C-NH) was prepared and tested in vivo for its safety, sustainability, and effects as a collagen stimulator.

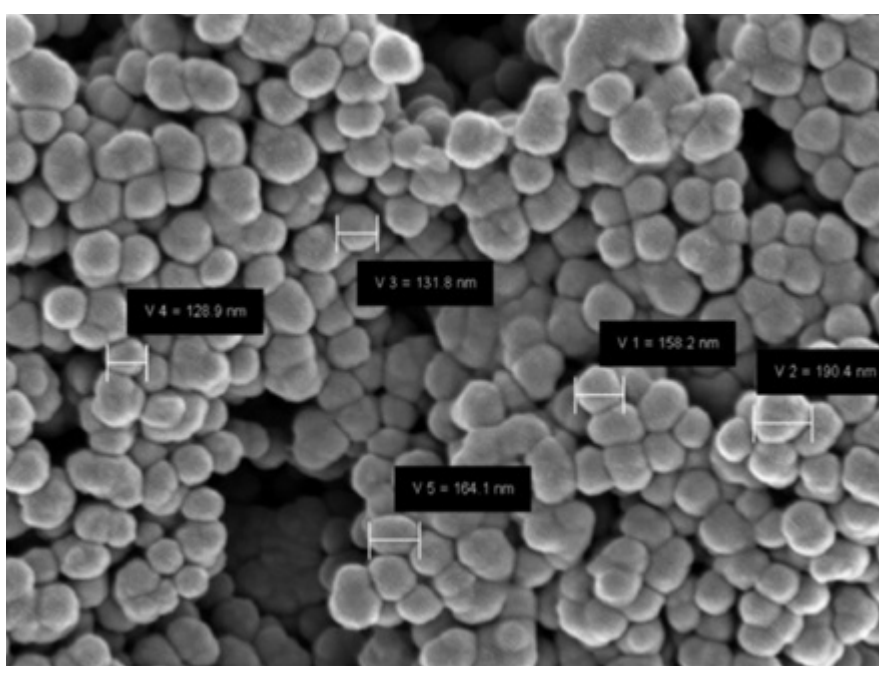

Fig. 1. Scanning electron microscopy image of the nano-sized polycaprolactone dermal filler preparation for the experiment $(\times 100,000)$. The average particle size is $200 \mathrm{~nm}$, and the particles are spherical.

\section{METHODS}

This study was carried out in the animal laboratory (Hallym-2010-78) of the Burn Research Center, Hangang Sacred Heart Hospital by the Department of Plastic and Reconstructive Surgery, Gangdong Sacred Heart Hospital in cooperation with Rose Plastic Surgery Clinic and Rose Lab.

Conventional PCL-based dermal fillers usually consist of about $30 \%$ (by weight) of PCL microspheres, and the fillers are homogenously suspended in aqueous-based CMC gel. PCL-C-NH was the material used in this experiment. It has an average particle size of $200 \mathrm{~nm}$ and is spherical and hydrophilic (Fig. 1). PCL-C-NH is a PCL-based polymer that has a small particle size with the property of resisting phagocytosis by aggregation of particles in vivo using sol-gel transition. The interpretation of the final results of cytotoxicity testing using the agar diffusion method was carried out according to the Ministry of Food and Drug Safety Notice No. 2014115 and Common Standards and Specifications for Biological Safety of Medical Devices, ISO 10993-5 (Table 1). No discoloration zone was observed in the middle layer of the cells to which the three test substances were administered, and no degree of cytolysis was ob-

Table 1. Degrees of cytotoxicity in the agar diffusion test

\begin{tabular}{lll}
\hline Degree & Reactivity & Description of reactivity zone \\
\hline 0 & None & No detectable zone around or under specimen \\
1 & Slight & Some malformed or degenerated cells under specimen \\
2 & Mild & Zone limited to area under specimen \\
3 & Moderate & Zone extending from specimen, with a size up to $1.0 \mathrm{~cm}$ \\
4 & Severe & Zone extending farther than $1.0 \mathrm{~cm}$ beyond specimen \\
\hline
\end{tabular}

Table 2. Results of the cytotoxicity (agar) test

\begin{tabular}{lcll}
\hline Test & Percent lysis & Reactivity & Grade \\
\hline Test sample & & & \\
(1) & 0 & None & 0 \\
(2) & 0 & None & 0 \\
(3) & 0 & None & 0 \\
(4) & 0 & None & 0 \\
Negative control ${ }^{\text {a) }}$ & & & \\
PCL-C-HN (1) & 0 & None & 0 \\
PCL-C-HN (2) & 0 & None & 0 \\
Positive control ${ }^{\text {b) }}$ & & & \\
Physiological saline (1) & 100 & Moderate & 3 \\
Physiological saline (2) & 100 & Moderate & 3 \\
\hline
\end{tabular}

${ }^{a}$ Negative control: with polycaprolactone copolymer nanoparticle hydrogel (PCL-C-NH) as the specimen, $30 \mu \mathrm{g}$ of $\mathrm{N}$-methyl-N-nitro-N-nitrosoguanidine (NTG) was immersed per sheet of round filter paper (diameter: $8 \mathrm{~mm}$ ); ${ }^{\text {bl Pos- }}$ itive control: physiological saline was immersed in NTG filter paper. 
served in the lower part of the specimen. Therefore, the test substance can be regarded as having no reactivity to cultured cells in this test environment. A reactivity zone was not observed in the middle layer of the solvent control group or negative control group, and a reactivity zone of $0.5 \mathrm{~cm}$ or more was observed in the positive control group. Under the test conditions, the PCL nanoparticle hydrogel did not induce dissolution or toxicity in mouse fibroblasts (Table 2).

The in vivo experiment was carried out on 36 Sprague-Dawley (SD) rats. Each animal weighed roughly $194.2 \mathrm{~g}$ and was an average of 6 weeks old at the beginning of the experiment. Animals were individually numbered on the tail and housed in plastic cages placed in ventilated racks at $24^{\circ} \mathrm{C}$ and $56 \%$ moisture in a 12 -hour light-dark cycle.

The SD rats were divided into three groups for the experiment. Group A ( $\mathrm{n}=12)$ was injected with normal saline (JW Pharma, Seoul, Korea). Group B $(n=12)$ was injected with PCL dermal filler (Ellanse M; JW Pharma). Group C $(n=12)$ was injected with PCL-C-NH (Rose Lab, Seoul, Korea).

Before the injections, each rat was anesthetized with isoflurane
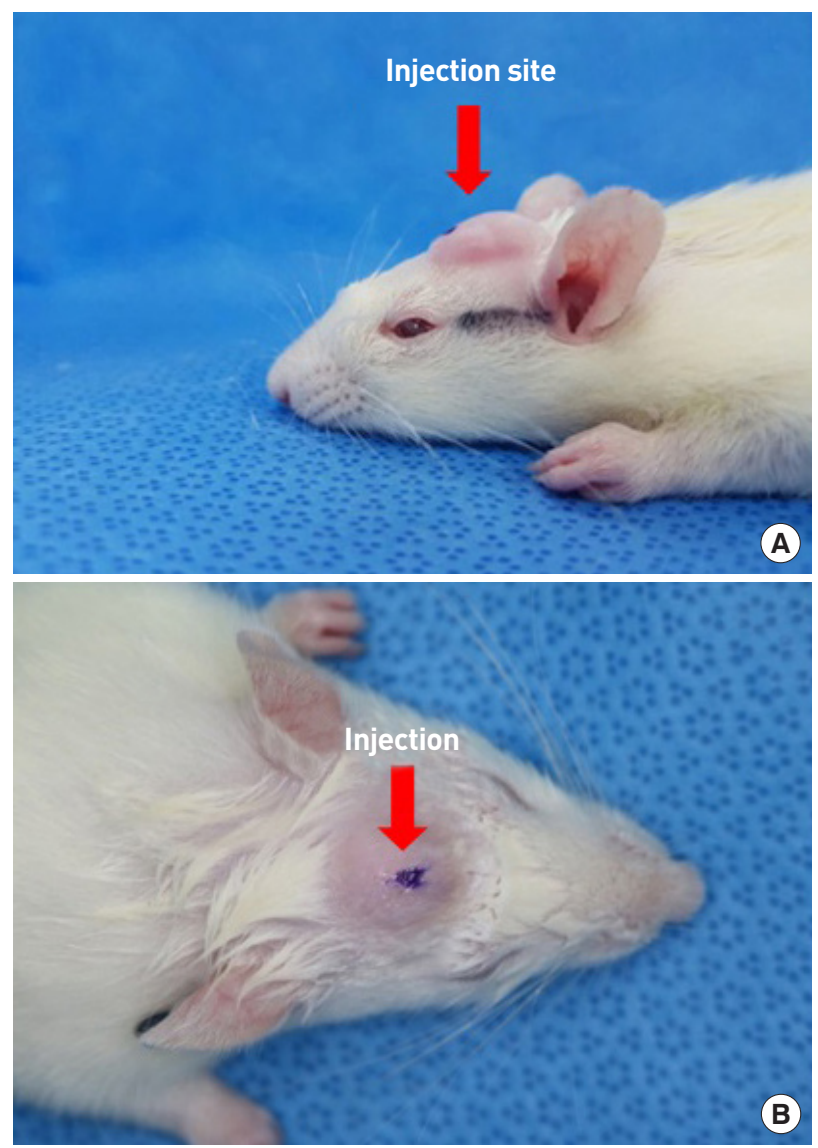

Fig. 2. Sprague-Dawley rat scalp after polycaprolactone copolymer nanoparticle hydrogel (PCL-C-NH) injection. Arrows indicate the injection site. (A) Left view. (B) Upper view. gas (Hana Pharm. Co., Ltd., Hwaseong, Korea). The animal was placed on a surgical table in the prone position, and its paws were tied with elastic strips. A $2 \times 1-\mathrm{cm}$ surface of the rat's scalp was depilated.

The fanning technique was used to inject $0.1 \mathrm{~mL}$ of material into the scalp dermal layer with uniform pressure using a disposable insulin syringe (26 gauge) (Fig. 2).

Four SD rats in each group (A, B, and C) were sacrificed at week 4 , week 8 , and week 12. Blood samples were obtained and used for a complete blood count (CBC) analysis and studies of liver and kidney function. For the histological examination, the liver and kidney tissue were fixed in $4 \%$ formaldehyde, embedded in frozen section media (Surgipath FSC22 clear; Leica Biosystems, Wetzlar,
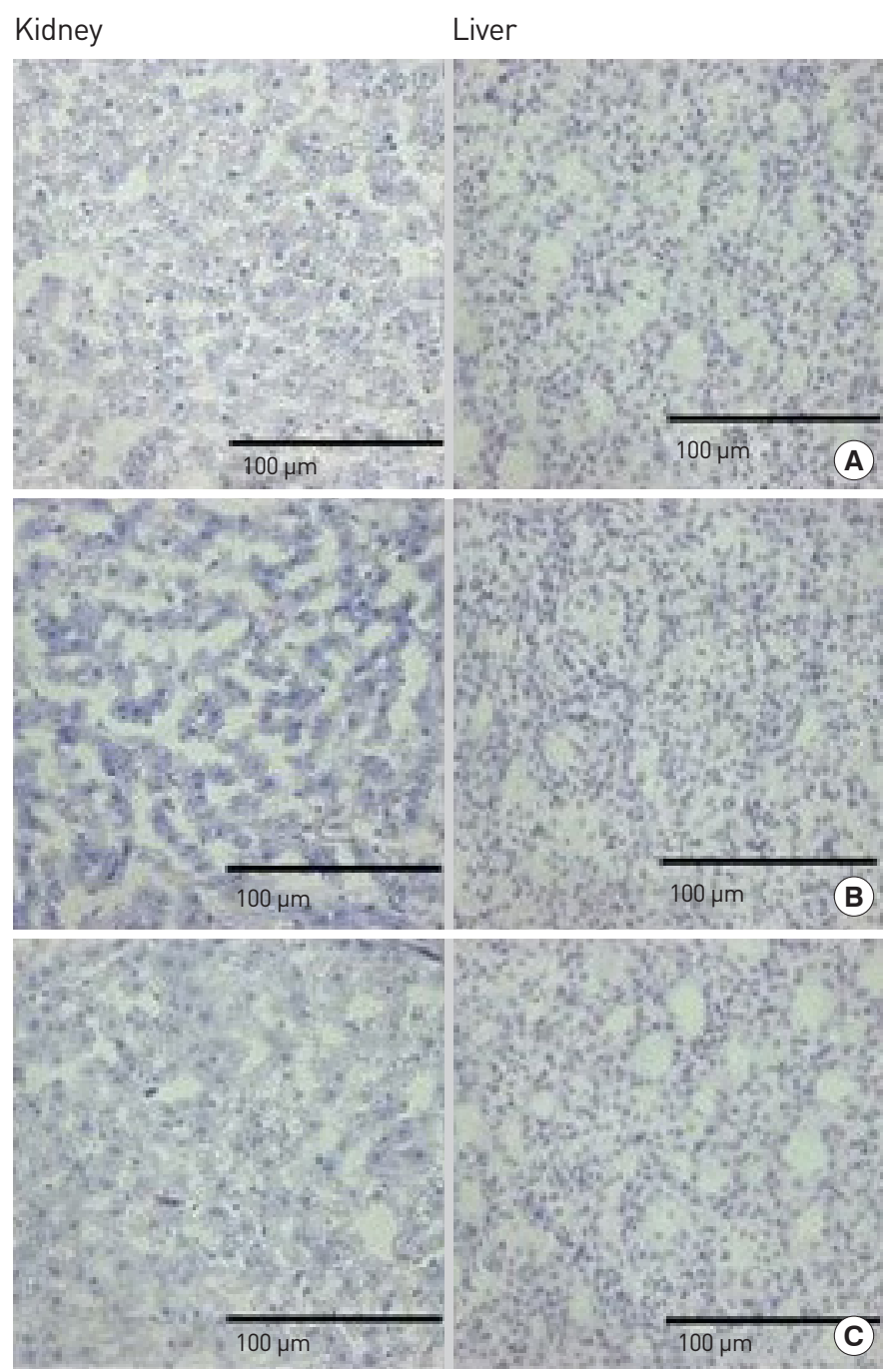

Fig. 3. Photographs of the rat liver and kidneys at 12 weeks $(H \& E$, $\times 400$ ). No tissue infiltration was found in either the group injected with the polycaprolactone (PCL) dermal filler or the group injected with the PCL copolymer nanoparticle hydrogel (PCL-C-NH), when compared to the control group injected with saline (scale bars $=100$ $\mu \mathrm{m}$ ). (A) Saline, (B) PCL dermal filler, and (C) PCL-C-NH. 
Germany), and processed for hematoxylin and eosin staining $(\times 400)$. Five randomly selected fields from each slide were viewed by an observer to assess microvessel formation, cystic degeneration, fibrotic cicatrization, and infiltration of inflammatory cells.

For the collagen formation examinations, the dermal tissue of the scalp at the injection site was resected, including the marginal non-injected site, and was fixed in 4\% formaldehyde, embedded in frozen section media, and processed for Masson trichrome staining. In order to quantify the collagen formation induced by the injection of the test materials, the tissue at the site of the injection was isolated and accurately weighed. The tissue was then placed in a $0.5 \mathrm{M}$ acetic acid solution, at a 1/10 ratio of weight to volume, and stirred at $4^{\circ} \mathrm{C}$ for 18 hours. Next, the samples were centrifuged at $15,000 \times g$ for 60 minutes, and $100 \mu \mathrm{L}$ of supernatant was collected. After adding $1 \mathrm{~mL}$ of the Sircol dye reagent, the collagen sample
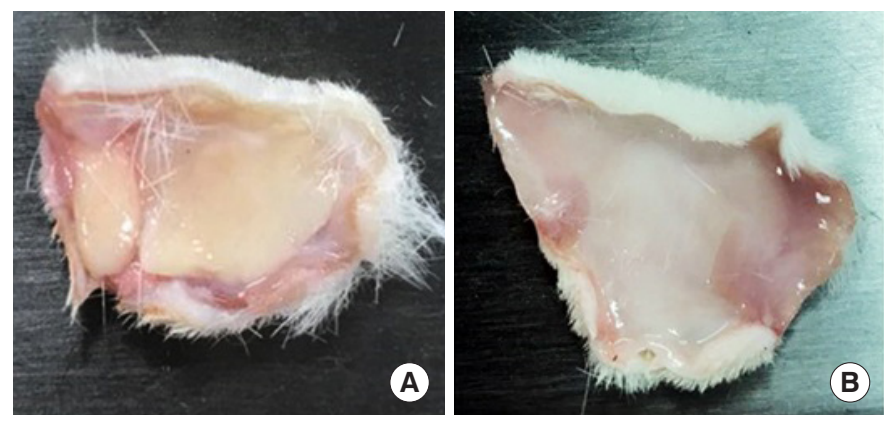

Fig. 4. Gross findings of the Sprague-Dawley rat scalps at 12 weeks. (A) Existing polycaprolactone (PCL) dermal filler (microspheres) and (B) PCL copolymer nanoparticle hydrogel (PCL-C-NH). Lump formation was observed in $(A)$ the conventional dermal filler, while no lump formation was observed in $(B)$ the scalp tissues injected with PCL-C-NH.

Fig. 5. Masson trichrome staining of SpragueDawley rat scalps at 12 weeks $(\times 100)$. (A) Saline, (B) PCL dermal filler, and (C) PCL-C-NH. No collagen formation was observed after $(A)$ injection with saline, whereas injections with (B) the PCL dermal filler and (C) PCL-C-NH induced collagen fiber formation, as shown by the arrows in enlarged crosssections (scale bars=100 $\mu \mathrm{m}$ ). PCL-C-NH, polycaprolactone copolymer nanoparticle hydrogel.
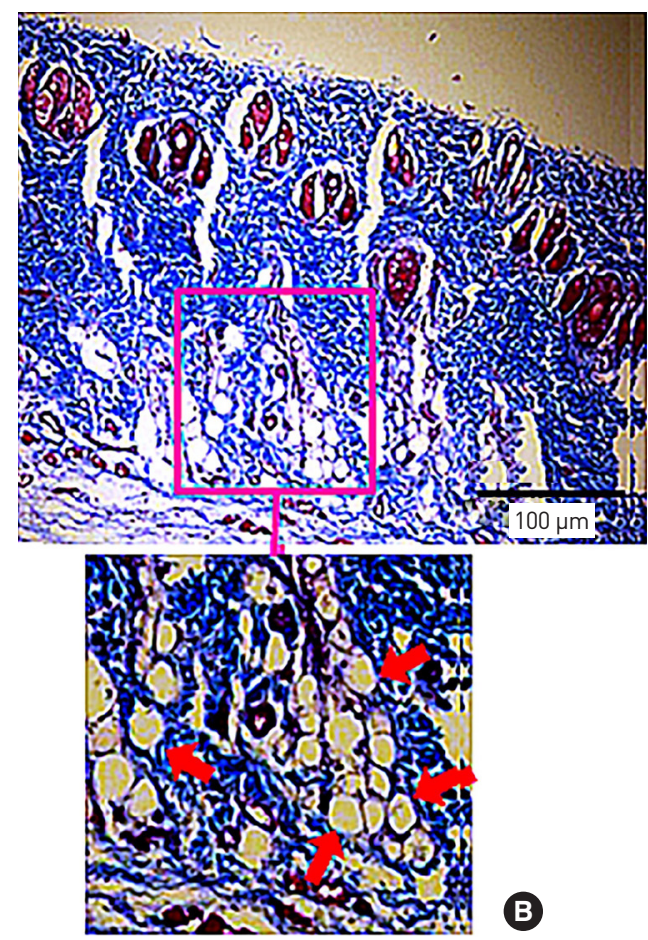

B

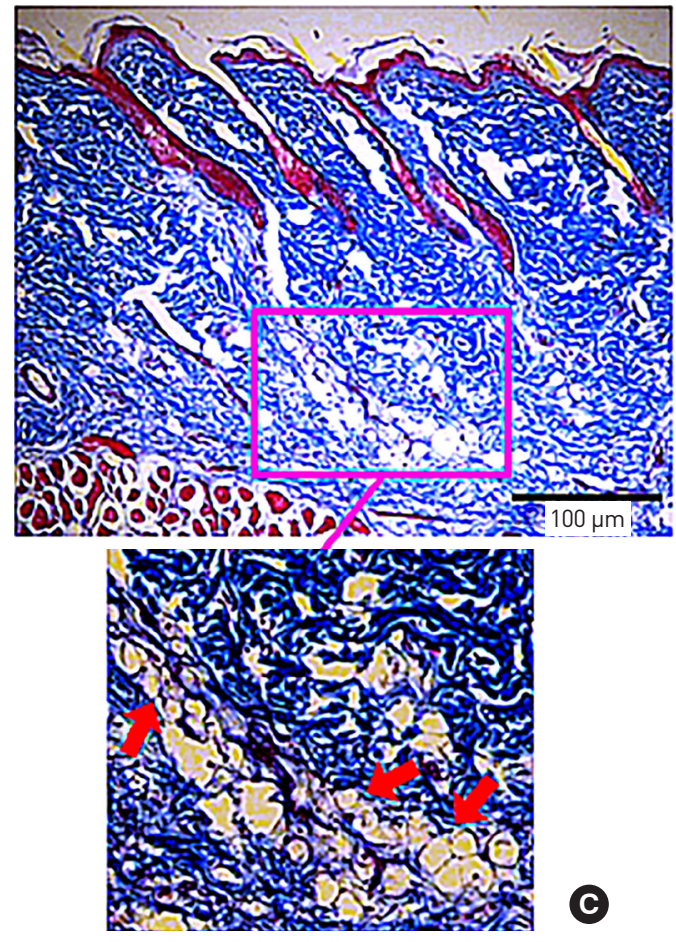


Table 3. Complete blood count analysis

\begin{tabular}{|c|c|c|c|c|c|c|c|c|}
\hline Group & WBC $\left(10^{3} / \mathrm{mm}^{3}\right)$ & $\operatorname{RBC}\left(10^{3} / \mathrm{mm}^{3}\right)$ & $\mathrm{Hb}(\mathrm{g} / \mathrm{dL})$ & Hct $(\%)$ & $\operatorname{MCV}\left(\mu \mathrm{m}^{3}\right)$ & $\mathrm{MCH}(\mathrm{pg})$ & $\mathrm{MCHC}(\mathrm{g} / \mathrm{dL})$ & $\operatorname{PLT}\left(10^{3} / \mathrm{mm}^{3}\right)$ \\
\hline B (microsphere filler) & $6.71 \pm 1.02$ & $8.14 \pm 0.24$ & $14.7 \pm 0.7$ & $44.8 \pm 0.6$ & $55.1 \pm 1.1$ & $17.4 \pm 0.4$ & $31.6 \pm 2.4$ & $966 \pm 8.87$ \\
\hline
\end{tabular}

WBC, white blood cell; RBC, red blood cell; $\mathrm{Hb}$, hemoglobin; Hct, hematocrit; MCV, mean corpuscular volume; $\mathrm{MCH}$, mean corpuscular hemoglobin; $\mathrm{MCHC}$, mean corpuscular hemoglobin concentration; PLT, platelet; PCL-C-NH, polycaprolactone copolymer nanoparticle hydrogel.

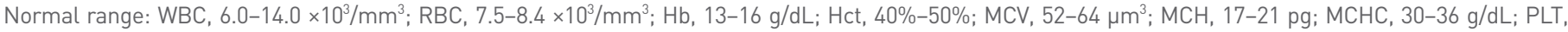
$900-1,200 \times 10^{3} / \mathrm{mm}^{3}$. No significant differences were observed in the results of the Kruskal-Wallis test $(P>0.05)$.

Table 4. Liver and kidney toxicity

\begin{tabular}{lcccc}
\hline Group & OT (UI/L) & PT (UI/L) & BUN (mg/dL) & $\mathrm{Cr}(\mathrm{mg} / \mathrm{dL})$ \\
\hline A (saline) & $37 \pm 4.28$ & $111 \pm 27.94$ & $12.7 \pm 2.51$ & $0.21 \pm 0.04$ \\
B (microsphere filler) & $33 \pm 4.94$ & $108 \pm 18.98$ & $17.1 \pm 2.27$ & $0.36 \pm 0.03$ \\
C (PCL-C-NH) & $33 \pm 6.27$ & $165 \pm 21.95$ & $14.7 \pm 2.68$ & $0.28 \pm 0.05$ \\
\hline
\end{tabular}

OT, oxaloacetic transaminase; PT, pyruvic transaminase; BUN, blood urea nitrogen; $\mathrm{Cr}$, creatinine; PCL-C-NH, polycaprolactone copolymer nanoparticle hydrogel.

Normal range: OT, 20-40 UI/L; PT, 80-200 UI/L; BUN, 11-16 mg/dL; Cr, 0.2$0.4 \mathrm{mg} / \mathrm{dL}$. No significant differences were observed in the results of the Kruskal-Wallis test $(P>0.05)$.

and staining reagent were mixed and centrifuged at $10,000 \times g$ for 10 minutes. One milliliter of the alkali reagent was then added to the pellet, and the collagen amount in each tissue was quantitatively measured using the soluble collagen assay kit (Sircol; Biocolor Ltd., Belfast, Ireland), which measures absorbance at $540 \mathrm{~nm}$.

Statistical analyses were performed using SPSS version 22.0 for Windows (IBM Corp., Armonk, NY, USA). The results of the three groups were compared using the Kruskal-Wallis test. A P-value of $<0.05$ was considered to indicate statistical significance.

\section{RESULTS}

Tissue infiltration of the particles was not observed in group $\mathrm{B}$ or group C, as compared with group A (Fig. 3).

The gross findings of scalp dermal tissue in group $\mathrm{A}$ and group B showed a distinct pattern (Fig. 4). In group B, lumps were observed in nine out of the 12 rats for 12 weeks; the mean size was about $1.89 \times 1.62 \mathrm{~cm}$, and there was no noticeable difference with time. We believe that the lump caused side effects, such as nodule formation [15]. In group C, a thin lamellar-shaped layer was observed in all SD rats. Masson trichrome staining was performed to identify collagen formation in the dermis of the scalp in each group. In groups $\mathrm{B}$ and $\mathrm{C}$, collagen formation was observed, and collagen fibers were spread widely around the particles (Fig. 5).

No significant differences from the reference values were observed in terms of the $\mathrm{CBC}$, oxaloacetic transaminase levels, pyruvic transaminase levels, or the blood urea nitrogen to creatinine ra-

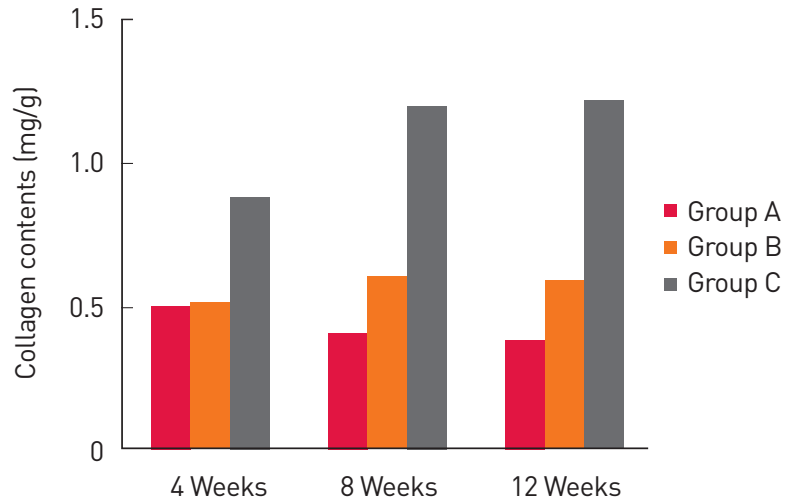

Fig. 6. Collagen quantification via the soluble collagen assay kit at 4 , 8 , and 12 weeks. Group A showed almost no change in collagen formation over time. Collagen formation in group $B$ slightly increased from $0.51 \mathrm{mg} / \mathrm{g}$ at week 4 to $0.6 \mathrm{mg} / \mathrm{g}$ at week 8 , with a small decrease from week 8 to week 12. Meanwhile, collagen in group $\mathrm{C}$ slightly increased by $0.34 \mathrm{mg} / \mathrm{g}$, from $0.88 \mathrm{mg} / \mathrm{g}$ at week 4 to 1.22 $\mathrm{mg} / \mathrm{g}$ at week 12. Group A, saline; group B, polycaprolactone (PCL) dermal filler; and group C, PCL copolymer nanoparticle hydrogel (PCL-C-NH).

tio when the three groups were compared. Furthermore, no significant differences were observed in the results of the Kruskal-Wallis test among the three groups $(\mathrm{P}>0.05)$. This suggests that the materials did not affect the hematopoietic system, the liver, or the kidneys (Tables 3,4 ).

Group A showed no changes in collagen formation. However, a slight reduction in collagen was observed in group A over time. In group B, soluble collagen slightly increased from $0.51 \mathrm{mg} / \mathrm{g}$ at week 4 to $0.6 \mathrm{mg} / \mathrm{g}$ at week 8 and remained at $0.6 \mathrm{mg} / \mathrm{g}$ through week 12 . Meanwhile, in group C, collagen formation slightly increased by $0.34 \mathrm{mg} / \mathrm{g}$, from $0.88 \mathrm{mg} / \mathrm{g}$ at week 4 to $1.22 \mathrm{mg} / \mathrm{g}$ at weeks 8 and 12 (Fig. 6).

\section{DISCUSSION}

PCL is known to be a biodegradable collagen stimulator that promotes neocollagenesis. In this study, nano-sized PCL copolymers were prepared in hydrogels to reduce the side effects caused by size 
and to stimulate collagen synthesis. The reason for this approach is that particles with a reduced size are easily injected, without any adverse effects, into the papillary dermis where relatively many fibroblasts are present.

Our results from animal experiments indicated that the material did not show any toxicity or tissue infiltration. Additionally, nanoparticles were aggregated with each other at the injection site, promoting collagen formation around them.

The assessment of collagen formation in the scalp tissues of SD rats through Masson trichrome staining indicated formation of collagen fibers around the tissues of the scalp skin in the PCL-C$\mathrm{NH}$ group. We observed more collagen formation in the PCL-C$\mathrm{NH}$ group than in the micro-sized PCL group.

Various fillers, such as HA or polymethylmethacrylate filler, have previously been found to be safe and effective [16,17]. Research on PCL dermal fillers is becoming more common. Some pilot studies have shown that PCL-based dermal fillers function as collagen stimulators, unlike conventional fillers. Additionally, PCL-based fillers have been shown to be safe, and patient satisfaction is high [7].

CMC molecules in the PCL dermal filler do not cross-link with each other, and the gel plays an important role in obtaining thinning properties [18]. These properties make the PCL-based dermal filler smooth, with an acceptable extrusion form during application. After injecting the filler, the CMC gel carrier is gradually absorbed by macrophages within several weeks, during which time the PCL microspheres promote neocollagenesis. Therefore, PCLbased dermal filler has almost the same elastic modulus as the dermis after neocollagenesis, but provides a better cosmetic result than HA dermal filler. Furthermore, type I collagen is synthesized, which, unlike other synthetic materials, produces a natural result that is not significantly different from physiologically occurring textures $[7,18]$.

It has been reported that when PCL-C-NH is produced into hydrogel, a sol-gel transition occurs under various conditions $(\mathrm{pH}$, temperature, etc.) [19]. Although PCL-C-NH is maintained in sol form at room temperature, it changes to gel form when it is injected into the body. Because of this characteristic, nanoparticles vulnerable to phagocytosis gradually become coagulated in response to temperature changes during injection into the body, where they are resistant to phagocytosis.

In this study, some properties of the biodegradable polymers were investigated. However, the stability of the substances used in the experiment has not yet been established, which could lead to problems in clinical applications; therefore, this issue needs to be addressed in the future.

Due to limitations in the use of the experimental animals, only four animal results for each experimental case were analyzed using the Kruskal-Wallis test. Hence, P-values were only available for the blood analysis test. The rat tissue collagen analysis in this experiment did not include a statistical analysis. Although the sample size was small, and the follow-up period was short, we believe that PCL$\mathrm{C}-\mathrm{NH}$ can be a next-generation material with fewer side effects and better efficacy than conventional fillers.

In conclusion, PCL-C-NH maintained biocompatibility and biodegradable properties in common with conventional PCL dermal fillers. The nanoparticle size reduced the adverse effects, such as nodule formation, caused by conventional microspheres with a larger particle size. PCL-C-NH can be easily injected into the dermal layer and is considered to promote neocollagenesis. If neocollagenesis occurs well with PCL-C-NH, as we hypothesize it will, it may help to enhance skin texture. Patients desire enhanced skin texture as much as volume augmentation, which is a typical indication for fillers. Hence, many attempts have been made to achieve both enhanced skin texture and volume augmentation. We experimented with a polymer that had not been previously used in fillers, to test the possibility of attaining enhanced skin texture; however, additional research on various other agents is needed.

\section{NOTES}

\section{Conflict of interest}

No potential conflict of interest relevant to this article was reported.

\section{Ethical approval}

Animal care and experimental procedures were approved by the Institutional Animal Care and Use Committee of Hallym University (IACUC No. HMC 2017-4-0720-26).

\section{ORCID}

Seung Jun Lee

Woo Sung Lee

Chul Hoon Chung

\section{REFERENCES}

1. Kablik J, Monheit GD, Yu L, et al. Comparative physical properties of hyaluronic acid dermal fillers. Dermatol Surg 2009;35 Suppl 1:302-12.

2. Gold MH. Use of hyaluronic acid fillers for the treatment of the aging face. Clin Interv Aging 2007;2:369-76.

3. de Melo F, Nicolau P, Piovano L, et al. Recommendations for volume augmentation and rejuvenation of the face and hands with the new generation polycaprolactone-based collagen stimulator (Ellansé $\left({ }^{\circledR}\right)$ ). Clin Cosmet Investig Dermatol 2017;10:431-40.

4. Andre P. New trends in face rejuvenation by hyaluronic acid injections. J Cosmet Dermatol 2008;7:251-8.

5. Tierney EP, Hanke CW. Recent trends in cosmetic and surgical procedure volumes in dermatologic surgery. Dermatol Surg 2009;35:132433.

6. Hwang $\mathrm{CH}$. The analysis of calcium hydroxylapatite as a subdermal filler in the rat [master's thesis]. University of Ulsan, College of Medi- 
cine: Seoul; 2009.

7. Morhenn VB, Lemperle G, Gallo RL. Phagocytosis of different particulate dermal filler substances by human macrophages and skin cells. Dermatol Surg 2002;28:484-90.

8. Bae IH, Kim MS, Choi H, et al. Ischemic oculomotor nerve palsy due to hyaluronic acid filler injection. J Cosmet Dermatol 2018;17:1016-8.

9. Parulan MA, Sundar G, Lum JH, et al. A case report on dermal fillerrelated periorbital granuloma formation. Orbit 2019;38:169-72.

10. Ammala A. Biodegradable polymers as encapsulation materials for cosmetics and personal care markets. Int J Cosmet Sci 2013;35:113-24.

11. Kim JA, Van Abel D. Neocollagenesis in human tissue injected with a polycaprolactone-based dermal filler. J Cosmet Laser Ther 2015;17: 99-101.

12. Niezabitowska E, Smith J, Prestly MR, et al. Facile production of nanocomposites of carbon nanotubes and polycaprolactone with high aspect ratios with potential applications in drug delivery. RSC Adv 2018; 8:16444-54.

13. Stander BA, van Vollenstee FA, Kallmeyer K, et al. An in vitro and in vivo study on the properties of hollow polycaprolactone cell-delivery particles. PLoS One 2018;13:e0198248.
14. Khandaker M, Riahinezhad S, Sultana F, et al. Effect of collagen-polycaprolactone nanofibers matrix coating on the in vitro cytocompatibility and in vivo bone responses of titanium. J Med Biol Eng 2018;38: 197-210.

15. Chiang YZ, Pierone G, Al-Niaimi F. Dermal fillers: pathophysiology, prevention and treatment of complications. J Eur Acad Dermatol Venereol 2017;31:405-13.

16. Medeiros CC, Borghetti RL, Nicoletti N, et al. Polymethylmethacrylate dermal fillers: evaluation of the systemic toxicity in rats. Int J Oral Maxillofac Surg 2014;43:62-7.

17. Fernandez-Cossio S, Castano-Oreja MT. Biocompatibility of two novel dermal fillers: histological evaluation of implants of a hyaluronic acid filler and a polyacrylamide filler. Plast Reconstr Surg 2006;117:178996.

18. Nicolau PJ, Marijnissen-Hofste J. Neocollagenesis after injection of a polycaprolactone based dermal filler in a rabbit. Eur J Aesthet Med Dermatol 2013;3:19-26.

19. Matsumoto Y, Shundo A, Ohno M, et al. Evolution of heterogeneity accompanying sol-gel transitions in a supramolecular hydrogel. Soft Matter 2017;13:7433-40. 